Check for updates

Cite this: RSC Adv., 2019, 9, 24915

Received 4th July 2019

Accepted 2nd August 2019

DOI: $10.1039 / \mathrm{c} 9 \mathrm{ra05064g}$

rsc.li/rsc-advances

\section{Continuous production of lignin nanoparticles using a microchannel reactor and its application in UV-shielding films}

\author{
Ting Ju, ${ }^{a}$ Zhiliang Zhang, (D)*a Yang Li, ${ }^{a}$ Xinfeng Miao ${ }^{a}$ and Jianbing Ji (D) *ab
}

\begin{abstract}
With the severe energy and environmental issues, lignin has received increasing attention as a renewable biomass feedstock. The development of lignin-based nanoparticles provides a new route to the valorization of lignin. In this work, we propose a simple continuous method to prepare lignin nanoparticles (LNS) using a microchannel reactor. Polyvinylpyrrolidone (PVP)/sodium dodecyl sulfate (SDS) were selected as stabilizers. Spherical-like lignin nanoparticles with an average size of $13 \mathrm{~nm}$ were obtained in a T-shaped microchannel reactor. The effects of solvent species, PVP/SDS mass ratio, and lignin solution flow rate on the size of LNS were investigated. The as-prepared LNS had a good stability during 60 days-storage and were used as an additive to form UV-shielding composite films with poly(vinyl alcohol) (PVA). Compared with raw lignin, the addition of LNS could enhance the UV-shielding efficacy by $13.3 \%$ in the ultraviolet spectrum $(250 \mathrm{~nm}$ ). The present work suggests that the microchannel reactor is a promising continuous approach to prepare LNS with versatile applications.
\end{abstract}

\section{Introduction}

Lignin, the second most abundant renewable natural biopolymer after cellulose, has attracted intensive research interest in recent years. ${ }^{1}$ Annually, over 70 million tons of industrial lignin are produced as a byproduct of biomass separation processes. ${ }^{2}$ However, practical applications of lignin are usually relegated to low end applications such as combustion for the generation of electricity and/or heat, because of its complicated molecular structure. ${ }^{3}$ There is still a great opportunity for further research to explore more valuable applications.

The monomeric units of lignin include monolignols, coniferyl alcohol, sinapyl alcohol and $p$-coumaryl alcohol, ${ }^{4}$ which are linked together by aromatic, aliphatic ether bonds, or $\mathrm{C}-\mathrm{C}$ bonds. ${ }^{5}$ The abundance functional groups including methoxy, phenolic hydroxyl, etc. ${ }^{6}$ in the lignin structure make it a promising candidate for the formation of polymer composites. ${ }^{7}$ Recent research has indicated that lignin is a versatile filler for the preparation of various composite materials, such as thermosets, ${ }^{\mathbf{8}}$ thermoplastics, ${ }^{\mathbf{9}}$ elastomers, ${ }^{\mathbf{1 0}}$ and antioxidants. ${ }^{\mathbf{1 1}}$ Considering the outstanding UV shielding properties of the phenolic hydroxyl groups, lignin is also a biodegradable alternative for producing UV-absorbing nanocomposites. ${ }^{12}$

${ }^{a}$ College of Chemical Engineering, Zhejiang University of Technology, Hangzhou 310014, China. E-mail: zhangzl@zjut.edu.cn; jjb@zjut.edu.cn; Tel: +86-571-88320043 ${ }^{b}$ Zhejiang Province Key Laboratory of Biofuel, Biodiesel Laboratory of China Petroleum and Chemical Industry Federation, Zhejiang University of Technology, Hangzhou 310014, China
Poly(vinyl alcohol) (PVA), a nontoxic, water-soluble and biodegradable polymer, ${ }^{\mathbf{1 3}}$ has been widely used as a polymer matrix to prepare PVA/lignin composite films for UVabsorbing. ${ }^{14}$ The performance of PVA/lignin is highly dependent on the particle size of lignin. However, particle size of the commercial available lignin are usually in the range of several tens to even several hundreds of microns, which are too large and may introduce detrimental impact on properties of PVA/ lignin films, such as inherent heterogeneity and poor dispersibility. ${ }^{15}$ An effective strategy to solve this problem is transform large lignin particles into nanoparticles.

Lignin nanoparticles have been synthesized by various methods including liquid precipitation, ${ }^{\mathbf{1 6}}$ sonication, ${ }^{17}$ aerosol processing $^{18}$ and mechanical treatment. ${ }^{19}$ Among these, liquid precipitation is widely used to prepare lignin nanoparticles thanks to its convenient procedure, low-cost, and use of simple equipment. ${ }^{20}$ By using THF as solvent, Lievonen et al. ${ }^{2}$ prepared colloidal lignin nanoparticles. For the precipitation step, the solution was placed into a dialysis bag and immersed in excess of distilled water for at least $24 \mathrm{~h}$. The resulting lignin nanoparticles were spherical particles with an average diameter between 200 and $500 \mathrm{~nm}$. In 2014, acetylated spherical lignin nanoparticles with an average hydrodynamic radius of $110 \mathrm{~nm}$ were synthesized by Qian et al. ${ }^{\mathbf{2 1}}$ Lignin nanoparticles were prepared by adding dropwise deionized water to the lignin solution. The obtained lignin nanoparticles showed a separation of the nanoparticle's core and shell. In 2016, Richter et al. ${ }^{22}$ produced organosolv lignin nanoparticles with a diameter of $\sim 80 \mathrm{~nm}$. The prepared nanoparticles present a good stability at specific $\mathrm{pH}$ range. Although the liquid precipitation showed 
great potential for preparation of lignin nanoparticles, however, the precipitations are usually conducted in a discontinuous manner, where the local mixing environment is not well controlled, resulting mass transfer limitations and significant chemical fluctuations so that yield the non-homogeneous and large-sized nanoparticles. ${ }^{23,24}$

In recent years, microchannel reactors have been used in many fields due to their continuous production, excellent mixing performance and precise control of reaction conditions. ${ }^{25}$ Compared to conventional batch reactors, microchannel reactors provide a homogeneous reaction volume with precise concentration, temperature, and mass transfer, resulting in a better control of the particle size and its distribution. ${ }^{20,23,26}$ Microchannel reactors have shown great promise for continuous preparation of inorganic nanoparticles, ${ }^{27}$ biological materials ${ }^{28}$ and semiconductor nanoparticles of $\mathrm{CdSe}^{29}$ and $\mathrm{TiO}_{2}{ }^{30}$ and noble metal nanoparticles of $\mathrm{Au}^{31}$ and Ag. ${ }^{32}$ Nevertheless, relatively little research has been done to make use of the benefits of microchannel reactors for the production of polymer nanoparticles, especially lignin nanoparticles. ${ }^{25}$

In this work, we proposed an approach for continuous producing of lignin nanoparticles by using a microchannel reactor. In fact, due to the high surface energy, lignin nanoparticles always have a tendency to agglomerate automatically, which in turn would limit its' potential applications. To avoid this shortage, polyvinylpyrrolidone (PVP)/sodium dodecyl sulfate (SDS) was used as polymer/surfactant stabilizers to prevent the agglomeration of lignin nanoparticles. ${ }^{33}$ This PVP/ SDS stabilized lignin nanoparticles were used to form composite films with PVA. Due to the introduce of PVP, the obtained films showed enhanced dispersibility and inherent homogenization. ${ }^{34}$

\section{Experimental}

\section{Materials}

Alkali lignin was purchased from Hefei Bomei Biotechnology Co. Poly(vinyl alcohol) (PVA) (degree of saponification 92-94\%), poly(vinyl pyrrolidone) (PVP) K29 32, sodium dodecylsulfate (SDS) (99\% purity), methanol, tetrahydrofuran (THF), $\mathrm{N}, \mathrm{N}$ dimethylformamide (DMF), and dimethyl sulfoxide (DMSO) were provided by Aladdin Co. All reagents were of analytical grade and were used as received.

\section{Preparation of lignin nanoparticles}

In this study, a T-shaped microchannel reactor was used to prepare lignin nanoparticles (LNS) by liquid precipitation method. Fig. 1(a) shows a photograph of the T-shaped microchannel used. Parameters of the microchannel reactor are schematically shown in Fig. 1(b). A flow diagram of the precipitation process is shown in Fig. 1(c). The entire system consisted of a microchannel reactor, two nonpulsatile pumps for supplying the lignin solution and the antisolvent, and a module for collecting the LNS suspension.

In a typical experiment, $1.0 \mathrm{~g}$ lignin was dissolved in $100 \mathrm{ml}$ solvent (methanol/THF $=3 / 1$, volume ratio) to obtain lignin
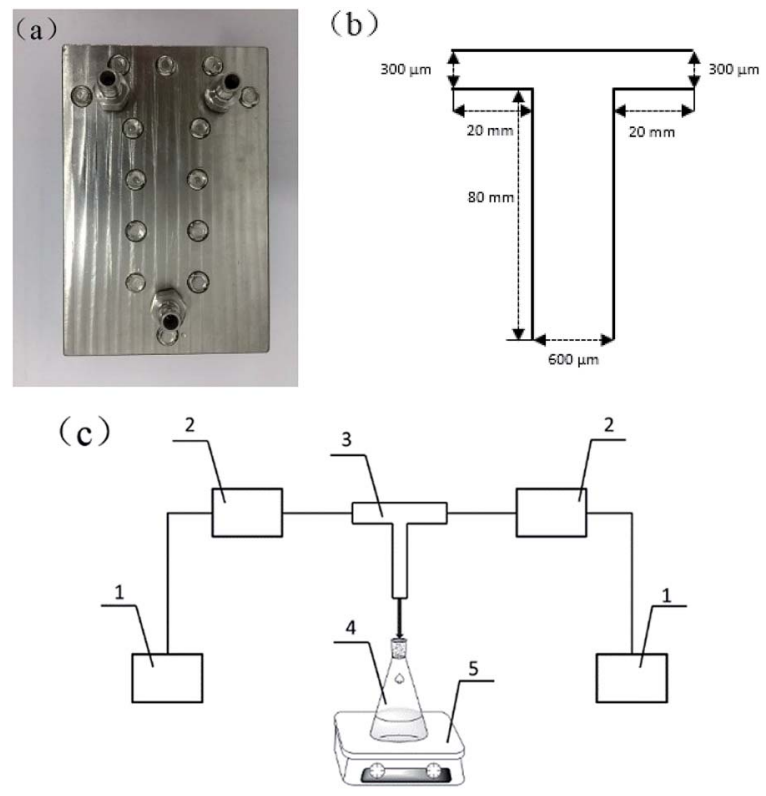

Fig. 1 (a) Picture of the T-shaped microchannel. (b) Schematic of the microchannel section. (c) Schematic diagram of the entire experimental setup: (1) tank, (2) nonpulsatile pumps, (3) T-shaped microchannel, (4) container, (5) stirred collection unit.

solution, while $4.0 \mathrm{~g}$ PVP and $0.1 \mathrm{~g}$ SDS were dissolved in $100 \mathrm{ml}$ deionized water (stabilizers solution). The lignin solution was then filtered through a $0.22 \mu \mathrm{m}$ syringe filter to remove undissolved impurities, and the concentration was calculated to be approximately $0.5 \%$. Subsequently, the lignin solution (1.0 $\mathrm{ml} \mathrm{min}{ }^{-1}$ ) was introduced into the T-typed microchannel to mix with another branch fluid of stabilizers solution. The effluent from the outlet stream was stirred at $300 \mathrm{rpm}$ for $10 \mathrm{~min}$ to obtain lignin nanosuspension for further use.

\section{Formation of nanocomposite films}

The nanocomposite films were prepared by a typical solution casting method. $20.0 \mathrm{~g}$ PVA was completely dissolved in $180 \mathrm{~g}$ deionized water and magnetically stirred for $3 \mathrm{~h}$ in a $95^{\circ} \mathrm{C}$ water bath. The PVA aqueous solution (10 wt $\%$ ) was then naturally cooled down to room temperature. 5.0 g PVA aqueous solution (10 wt\%) and a calculated amount of the LNS suspension prepared with different lignin solution flow rates $\left(1 \mathrm{ml} \mathrm{min}^{-1}, 2\right.$ $\mathrm{ml} \mathrm{min}{ }^{-1}, 3 \mathrm{ml} \mathrm{min}^{-1}, 4 \mathrm{ml} \mathrm{min}^{-1}, 5 \mathrm{ml} \mathrm{min}{ }^{-1}$ ) were mixed fully though magnetic stirring for $30 \mathrm{~min}$ to obtain a colloidal liquids, and the corresponding samples were named as P-P-S/ LNS-1，P-P-S/LNS-2， P-P-S/LNS-3， P-P-S/LNS-4， P-P-S/LNS-5, respectively. All samples were sonicated for $30 \mathrm{~min}$ to eliminate bubbles before being poured into polytetrafluoro ethylene dishes, and then dried at $70{ }^{\circ} \mathrm{C}$ in the vacuum oven for $24 \mathrm{~h}$ to remove solvents. For comparison, the pure PVA film, PVA film with raw lignin (RL) and the PVA film with PVP and SDS were prepared, and were named as pure PVA, P-P-S/RL and P-P-S, respectively. In each nanocomposite films, the mass ratio of lignin to PVA was all fixed at $1.0 \%$. Different nanocomposite films kept the same mass of lignin, PVP and SDS. The 


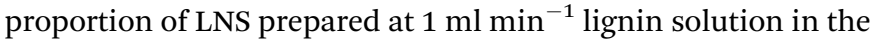
nanocomposite films was set at $0.5,1.0,1.5,2.0$ and $2.5 \mathrm{wt} \%$, and the corresponding sample was named P-P-S/0.5LNS, P-P-S/ 1.0LNS, P-P-S/1.5LNS, P-P-S/2.0LNS, P-P-S/2.5LNS, respectively.

\section{Characterization}

The mean particle size of LNS suspension was detected by dynamic light scattering (DLS) at $25^{\circ} \mathrm{C}$ with a Malvern Zetasizer Nano-ZS90 Instrument (UK). Scanning electron microscopy (SEM; Hitachi S-4700) and Transmission electron microscopy (TEM; Tecnai G2 F30) were used to observe the particle size and morphology of the samples. The optical transparency and UVshielding performance of the composite films were measured by PerkinElmer Lambda 35 UV-visible spectrophotometer. The scan speed was $240 \mathrm{~nm} \mathrm{~min}{ }^{-1}$. The thermal stability of nanocomposite films was evaluated by thermogravimetric analysis (TG) in TG209F3 (NETZSCH) at a high purity nitrogen flow rate of $50 \mathrm{ml} \mathrm{min}{ }^{-1}$. All the samples (3-4 mg) were equilibrated in the $\mathrm{Al}_{2} \mathrm{O}_{3}$ crucible at $30^{\circ} \mathrm{C}$, and then were heated up to $600{ }^{\circ} \mathrm{C}$ at a heating rate of $10^{\circ} \mathrm{C} \mathrm{min}^{-1}$.

\section{Results and discussion}

\section{Parameter vs. sizes of lignin nanoparticles}

The $\pi-\pi$ interactions and intermolecular hydrogen bonding make lignin difficult to solubilize in solvents, ${ }^{35,36}$ and meanwhile, the aliphatic hydroxyl groups of lignin can form strong hydrogen bonds with solvent molecules, ${ }^{35}$ which also decrease the solubility of lignin. On the other hand, the solubilization of lignin directly affects the formation of LNS., ${ }^{3,22,37}$ Therefore, preparation of LNS requires a careful selection of solvents. In this work, THF, methanol, DMSO and DMF were investigated. As shown in Fig. 2, at a fixed lignin solution/water flow rate ratio of $1: 10$, THF, methanol, DMSO and DMF give the average sizes of $\sim 850 \mathrm{~nm}, \sim 450 \mathrm{~nm}, \sim 400 \mathrm{~nm}$ and $850 \mathrm{~nm}$, respectively. It seems that DMSO is the suitable solvent for lignin. However, the poor solubility of lignin in DMSO is harmful to the yield of LNS. For THF and methanol, lignin has a good solubility,

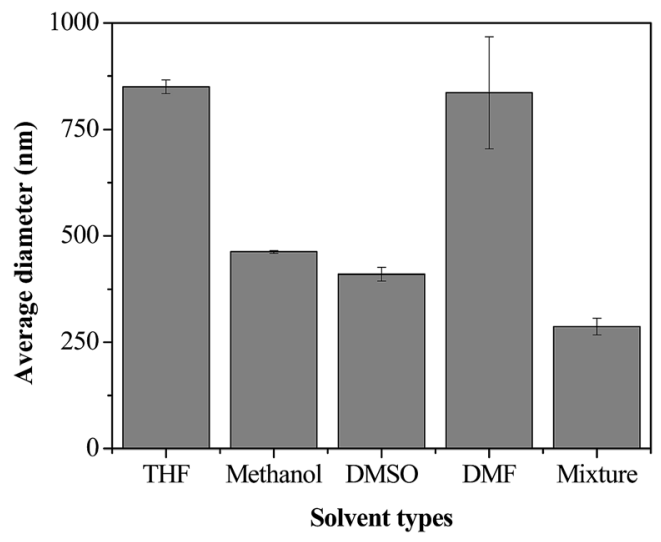

Fig. 2 Effect of the solvents type on the size of lignin nanoparticles (lignin solution flow rate $=1 \mathrm{ml} \mathrm{min}-1$; water flow rate $=10 \mathrm{ml} \mathrm{min}^{-1}$, for the methanol/THF mixture, methanol/THF volume ratio was $4: 1$ ). unfortunately, the large particle sizes of the obtained LNS makes them unfavorable for the preparation of LNS. It was reported that nanoparticles with much smaller size can be formed by the combination of solvents. ${ }^{38}$ Therefore, methanol/THF mixture was further evaluated, and as expect, a significantly smaller size of $290 \mathrm{~nm}$ was reached. As for DMF, due to the poor solubility of lignin and large size of LNS, it was not tested further.

To further investigate the effects of methanol/THF volume ratios, the lignin solution/water flow rate ratio was fixed at $1: 10$, while methanol/THF volume ratios was regulated to achieve $1: 1,2: 1,3: 1,4: 1$, and $5: 1$. As shown in Fig. 3(a), as the methanol/THF volume ratio increases from $1: 1$ to $3: 1$, the average diameter of LNS decreases to a minimum value of $270 \mathrm{~nm}$. However, further increasing methanol/THF volume ratio to $4: 1$ or $5: 1$, the average diameter increases to $287 \mathrm{~nm}$ and $340 \mathrm{~nm}$, respectively. This may be ascribed to the reasons that the polarity of methanol is superior to that of THF, and thus, increasing the ratio of methanol is favorable to the dissolution of lignin, which in turn increases the supersaturation level. According to the classical nucleation theory, with the increasing of the supersaturation level, the nucleation rate and particle growth rate was increased, while the ratio of particle growth rate to nucleation rate and the particle size exhibits a downward trend..$^{39,40}$ As a result, the average diameter of LNS was decreased with the increasing of methanol/THF volume ratio. However, due to the agglomeration of particles, further increasing the methanol/THF volume ratio leads to an increasing of particle size. Therefore, suitable stabilizers should be selected to prevent the agglomeration of LNS.

In this study, polyvinylpyrrolidone (PVP)/sodium dodecyl sulfate (SDS), one of the most widely used polymer/surfactant stabilizers for preparing pharmaceutical nanoparticles, was employed as stabilizers. $^{\mathbf{4 1 , 4 2}}$ As shown in Fig. 3(b), at different methanol/THF, the addition of PVP/SDS (mass ratio of $30: 1$ ) can significantly reduce the average size of LNS. At methanol/THF ratio of $3: 1$, a minimum size of $25 \mathrm{~nm}$ was reached, which is smaller by $\sim 10$ times than LNS prepared without PVP/SDS, indicating that PVP/SDS has a significant effect on particle size of LNS.

To further explore the effect of PVP/SDS mass ratio, the amount of SDS added into $100 \mathrm{~g}$ water was set at $0.1 \mathrm{~g}$. By
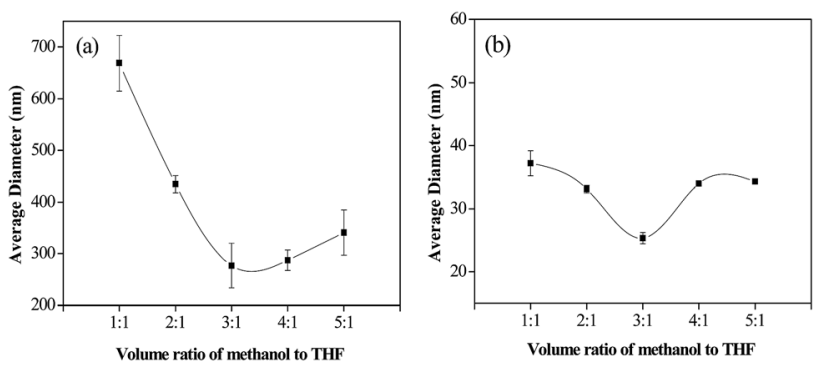

Fig. 3 (a) Effect of the methanol/THF ratio on the size of lignin nanoparticles (lignin solution flow rate $=1 \mathrm{ml} \mathrm{min}^{-1}$; water flow rate $=$ $10 \mathrm{ml} \mathrm{min}{ }^{-1}$ ); (b) effect of the methanol/THF ratio on the size of lignin nanoparticles in the presence of PVP/SDS (lignin solution flow rate $=1$

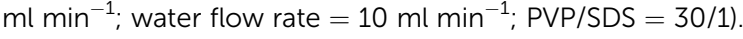


changing the amount of PVP, different PVP/SDS mass ratios were achieved. As shown in the Fig. 4, with the mass ratio of $\mathrm{PVP} / \mathrm{SDS}$ ranging from 1 to 40 , the average size of LNS decreased markedly from 250 to $13 \mathrm{~nm}$. After PVP/SDS mass ratio of 40, the average diameter was basically unchanged. In aqueous solution, the hydrocarbon tails of SDS molecules can bind at the methylene carbon centers of pyrrolidone rings of random-coiled PVP through hydrophobic interactions to form soft matter clusters. ${ }^{43}$ When PVP/SDS aqueous solution mixed with lignin solution, lignin micelles are formed due to the charging of supersaturation. The micelles are then immediately adsorbed by the soft matter clusters through hydrogen bonding, thus preventing the agglomeration of lignin micelles effectively and resulting a significant reduction of LNS. With the increasing of PVP, the soft clusters formed may reach at a threshold value, resulting almost all LNS completely adsorbed on soft clusters, therefore, the particle size was unchanged after PVP/SDS ratio of 40. According to the above investigations, the mass ratio of 40 was preferred as the optimal PVP/SDS ratio.

The flow rate ratio is a key factor which affects the size of nanoparticle in microchannel precipitation process. Changing the flow rate ratio can manipulate the size of the prepared nanoparticles. As shown in Fig. 5, increasing the flow rates of

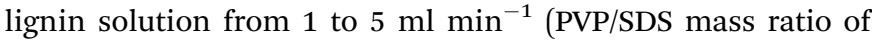
$40: 1$ ), the average diameters of LNS gradually increased from 13 to $32 \mathrm{~nm}$. The increase of LNS size could be explained by the formation rate of LNS and the adsorption capacity of soft clusters. It is known that the nucleation rate is positively correlated with the supersaturation. Increasing the flow rate of lignin solution leads to the increasing of supersaturation, and as a result, the lignin micelles formed are too much to be adsorbed by the PVP/SDS (40:1) soft matter clusters. Therefore, with PVP/SDS mass ratio of $40: 1$, the optimal lignin solution flow rate was $1 \mathrm{ml} \mathrm{min}^{-1}$.

A possible formation mechanism of the LNS was schematic shown in Scheme 1. When PVP/SDS solution mixed with lignin solution in the T-shaped microchannel, spherical lignin micelles were formed due to the changing of supersaturation ("Nucleation"). The micelles were then immediately adsorbed

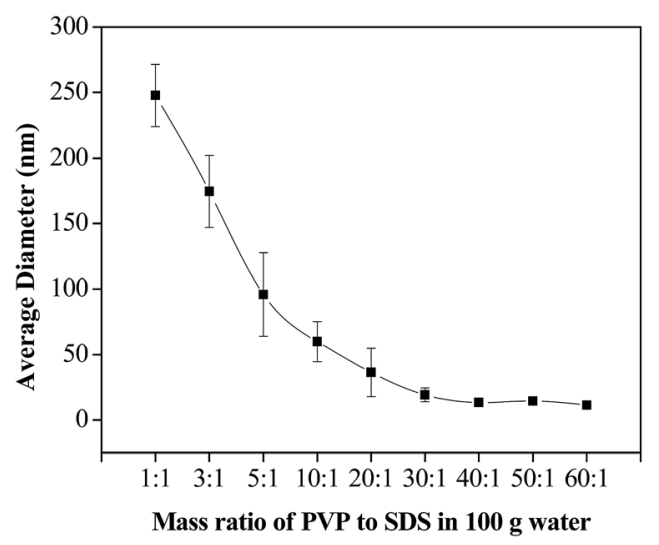

Fig. 4 Effect of PVP/SDS mass ratio on the size of lignin nanoparticles (methanol/THF $=3 / 1$; lignin solution flow rate $=1 \mathrm{ml} \mathrm{min}^{-1}$; water flow rate $=10 \mathrm{ml} \mathrm{min}{ }^{-1}$ ).

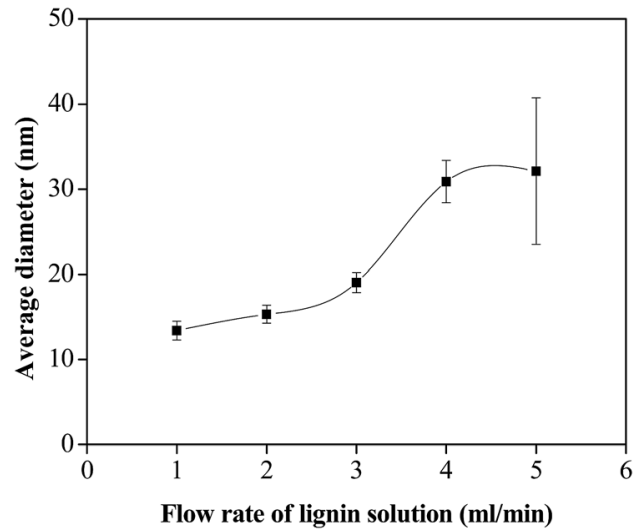

Fig. 5 Effect of the lignin solution flow rate on the size of lignin nanoparticles (methanol/THF $=3 / 1 ;$ PVP/SDS $=40 / 1$ ).

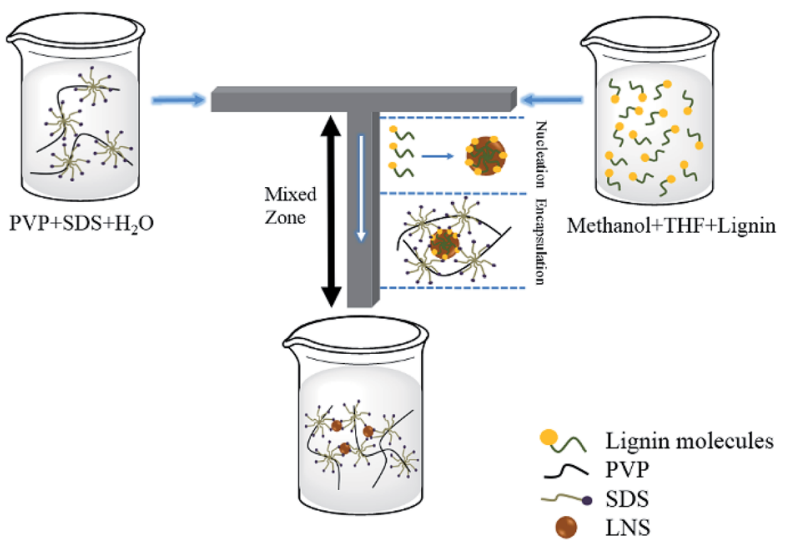

Scheme 1 A possible formation mechanism of lignin nanoparticles.

by the soft matter cluster ("Encapsulation"). As a result, LNS were successfully prepared by the microchannel reactor.

\section{Morphological characteristics and stability of the lignin nanoparticles}

The morphology of the prepared LNS and RL were compared by SEM and TEM. As shown in Fig. 6(a), (c) and (d), the LNS shows spherical-like shape and an average size of about 10-20 nm, matching well with the dynamic light scattering results. In contrast, the RL shows irregular shape and a board size distribution of $2-10 \mu \mathrm{m}$.

The storage stability of nanoparticle in suspension is a crucial concern for its applications. To evaluate the stability of LNS, the average size of the fresh prepared lignin nanosuspension was recorded with storage time. As shown in Fig. 7(a), the average size was kept at $13 \mathrm{~nm}$ during 60 days investigation. SEM observation reveals that the morphology and size of the LNS were the same as that of the fresh lignin nanosuspension, indicating the prepared LNS have a good stability.

\section{Application of the lignin nanoparticles in PVA film}

Morphological characteristics of the PVA films. In the work, the LNS stabilized by PVP/SDS were used to form PVA composite 


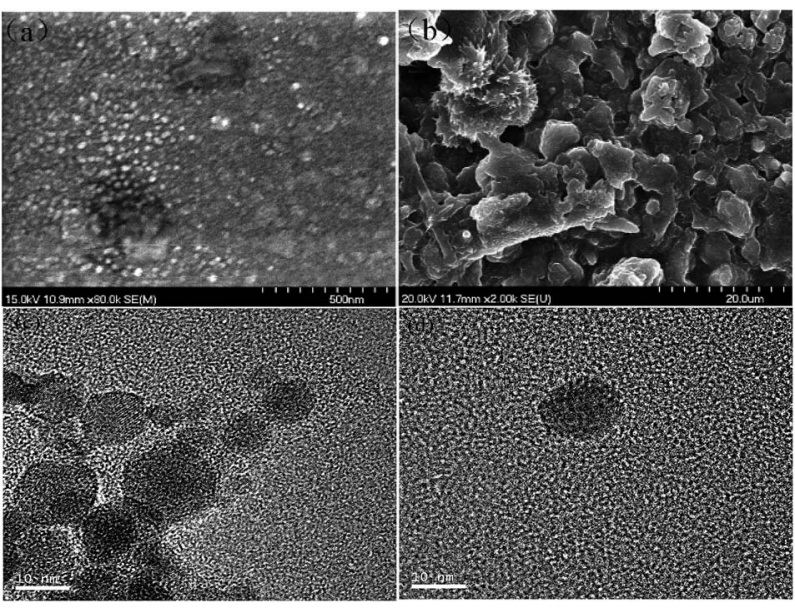

Fig. 6 SEM images of (a) the fresh LNS, and (b) the RL; TEM images of (c), (d) the fresh LNS

films. In order to confirm the compatibility of nanocomposites, the fracture surfaces of the pure PVA film, P-P-S film, P-P-S/LNS1 film, and P-P-S/RL film were characterized by SEM. As shown in Fig. 8, the pure PVA film and P-P-S film both exhibit a very smooth fracture surface. Neither gaps nor cracks were observed, indicating that the incorporation of PVP/SDS in PVA matrix doesn't have a negative impact on the compatibility of composite films. Fig. 8(c) shows the SEM of the P-P-S/LNS-1 film, the smooth and homogeneous surface indicated that the LNS stabilized PVP/SDS were dispersed homogeneously in the PVA matrix. In comparison, the P-P-S/RL film formed by RL shows a relatively matte surface, indicating that the RL had a poor compatibility with the polymer matrix. Similar results were observed by Xiao-Qin $\mathrm{Hu}$ et al., who prepared PVA composite films using RL. ${ }^{44}$ Due to the large particle size of lignin, the deteriorated compatibility was reported. ${ }^{45-47}$

Thermal stability (TG analysis) of the PVA films. TG and DTG curves of pure PVA, P-P-S/LNS-1, P-P-S/RL and P-P-S nanocomposites were recorded to study the effect of LNS on the thermal degradation behavior of films. As shown in Fig. 9(a), as followed by the entire decomposition of the composites, all the samples had three major weight loss stages. The first weight loss started at $80-120^{\circ} \mathrm{C}$ and corresponded to the exhaustion of the moisture from the polymer matrix and/or water molecules absorbed from the air. This weight loss was same to those of
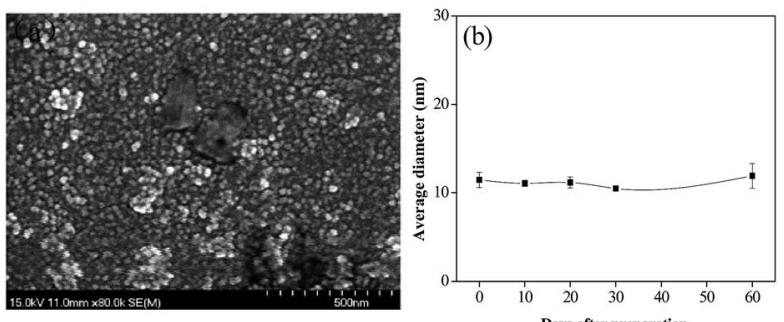

Fig. 7 (a) SEM images of LNS after 60 days-storage, and (b) the average hydrodynamic diameter of LNS as a function of time.

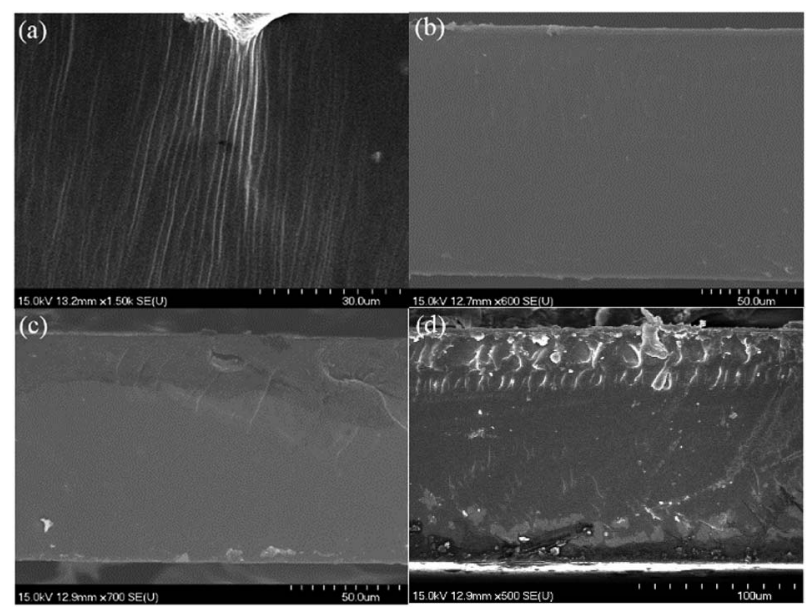

Fig. 8 SEM images of (a) the pure PVA film, (b) P-P-S film, (c) P-P-S/ LNS-1 film, and (d) P-P-S/RL film.

PVA, PVP, and lignin previously reported. ${ }^{48}$ The second weight loss stage was observed over a wide temperature range from 230 to $450{ }^{\circ} \mathrm{C}$. This weight loss was mainly related to the decomposition of acetate and hydroxyl groups of PVA and lignin. Compared to pure PVA, P-P-S/LNS-1, P-P-S/RL and P-P-S had the lower onset degradation temperatures $\left(T_{\text {onset }}\right)$, which could be attributed to the fact that the degradation of lignin and PVP start at a relatively lower temperatures than that of PVA. ${ }^{14}$ The $50 \%$ weight loss temperature of P-P-S film is at $373{ }^{\circ} \mathrm{C}$, which was higher than that of the pure PVA $\left(345^{\circ} \mathrm{C}\right)$, indicating that the thermal stability of PVA membrane can be improved by the addition of PVP/SDS. ${ }^{49}$ Interestingly, with the introduction of RL and LNS, the $50 \%$ weight loss temperature reached to $380{ }^{\circ} \mathrm{C}$ and $388^{\circ} \mathrm{C}$, respectively, which means that the thermal stability of the composite films can be further improved by lignin. It is attributed to the strong hydrogen bonds formed between the aromatic structures of lignin and PVA. ${ }^{44}$ The weight loss was observed in the TG spectrum of the PVA below $400^{\circ} \mathrm{C}$ indicated the decomposition of the PVA backbone structure. ${ }^{50}$ The third stage was ascribed to the fracture of the main chain of PVP and PVA, followed by the decomposition of polymer framework structure above $450{ }^{\circ} \mathrm{C}$. To sum up, LNS and RL could improve the thermal stability of PVA film, and P-P-S/LNS-1 performed better thermal stability than P-P-S/RL.

In Fig. 9(b), the maximum decomposition temperature $\left(T_{\max }\right)$ of pure PVA film $\left(320^{\circ} \mathrm{C}\right)$ was lower evidently than other
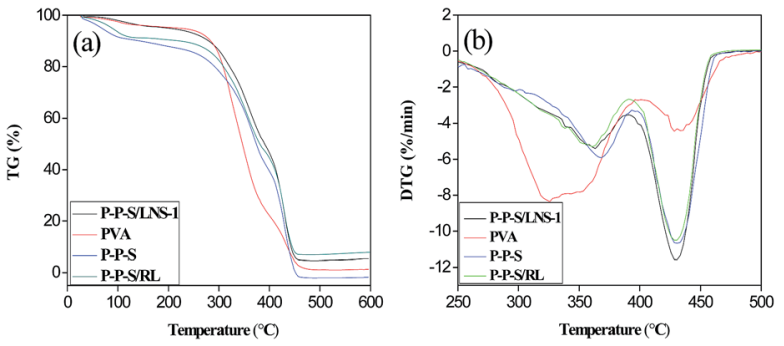

Fig. 9 TG (a) and DTG (b) curves of the nanocomposite films. 
samples. P-P-S/LNS-1 and P-P-S/RL had slightly lower $T_{\max }$ than P-P-S. A possible explanation for this phenomenon is that the $T_{\max }$ of lignin, PVA, and PVP followed the order: PVP $>$ lignin $>$ PVA. Additionally, the addition of LNS and RL decreased slightly the compatibility of PVA and PVP films. Considering the $T_{\max }$ improvement degree of the nanocomposite films compared to pure PVA films, the nanocomposite films prepared in this work were superior to the reported PVA/lignin composite films. ${ }^{\mathbf{1 4 , 1 9}}$ The variations of TG and DTG curves of nanocomposite films are similar to the reported films. ${ }^{51}$

UV-Vis absorbance of the PVA films. Fig. 10(a) compares the UV-Vis transmission of pure PVA film, P-P-S/LNS-1, P-P-S and P$\mathrm{P}-\mathrm{S} / \mathrm{RL}$ in the wavelength range from 200 to $800 \mathrm{~nm}$. For pure PVA and P-P-S film, ultraviolet light could not be blocked. Compared to the PVA and P-P-S, P-P-S/LNS-1 and P-P-S/RL showed strong absorption in the ultraviolet transmission spectra due to the existence of lignin. Moreover, it was found that the transmittance of P-P-S/LNS-1 was lower than that of P-P$\mathrm{S} / \mathrm{RL}$ in the region of the ultraviolet spectrum. This behaviour can be explained that the specific surface area of LNS is larger than that of the RL with the same mass. The functional groups such as phenolic hydroxyl and the basic phenylpropane unit for UV absorption act on the surface of the lignin particles. Hence, LNS has more anti-UV functional groups than RL with the same mass because of the difference in specific surface area, which resulting LNS performs better than RL for UV absorption.

The UV-Vis transmission spectrum of PVA nanocomposite films prepared with different lignin solution flow rates are presented in Fig. 10(b). In the visible spectrum region, all the nanocomposite films had high transmittance. There was no significant difference in the visible spectrum region. All the nanocomposite films showed strong absorption in the ultraviolet spectrum region, owing to the presence of LNS. Noted that
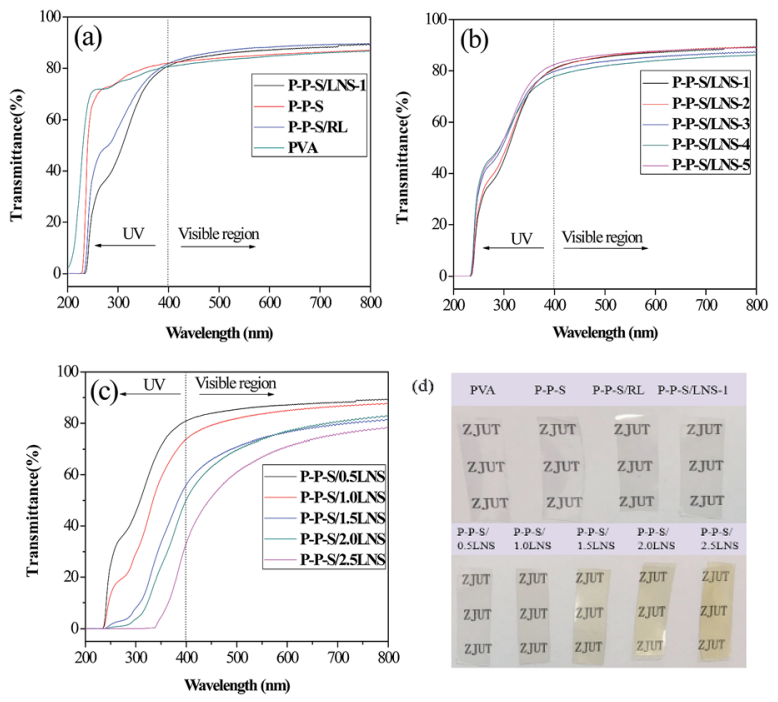

Fig. 10 (a) UV-Vis curves of pure PVA film, P-P-S/LNS-1, P-P-S and P$\mathrm{P}-\mathrm{S} / \mathrm{RL}$; (b) UV-Vis curves of the films obtained at different lignin solution flow rates; (c) UV-Vis curves of the films obtained at different LNS contents; (d) photographs of the films obtained at different conditions. the transmittance of PVA nanocomposite film prepared at the

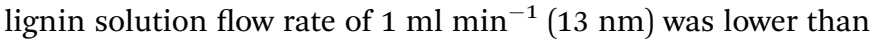
that of other films prepared at higher flow rate in the low wavelength region of the ultraviolet spectra, indicating smaller size nano-lignin exhibits greater UV resistance than larger size LNS. Therefore, it could be concluded that the UV resistance of nanocomposite films is controlled by the size of nano-lignin.

Fig. 10(c) shows the UV-Vis spectrum of PVA nanocomposite films with different LNS contents. Evidently, the films color slowly turns darker with increasing amount of LNS (Fig. 10(d)). With increasing of the LNS content, the ultraviolet-visible transmittance of the nanocomposite films decreased. This was because the nanocomposite films contained more LNS, and had the higher absorption rate of ultraviolet-visible light. In addition, the proportion of LNS in the nanocomposite films increased, which may result in the higher reflection and refraction of the light. ${ }^{52}$ In detail, the resulting nanocomposite ( $0.5 \mathrm{wt} \% \mathrm{LNS}$ ) retained $75.2 \%$ of UV irradiation in the ultraviolet spectrum (250 nm), without losing too much transparency, since $86.6 \%$ of the light transmittance in the visible light spectrum (550 nm) could be observed. When increasing LNS content to $2.5 \mathrm{wt} \%$, the UV light was almost completely shielded under $340 \mathrm{~nm}$, whereas the light transmittance at $550 \mathrm{~nm}$ reached only $66.8 \%$. The above results indicated that the addition of LNS to the PVA could improve efficiently anti-ultraviolet ability with slightly sacrificing its visible light transparency.

\section{Conclusions}

In this work, we demonstrated continuous precipitation of lignin nanoparticles using a T-shaped microchannel reactor. With PVP/SDS as stabilizers, LNS with an average size of $13 \mathrm{~nm}$ were prepared. The LNS exhibited good stability during 60 daysstorage and could be homogeneously dispersed in the PVA matrix. Compared with the raw lignin, the addition of LNS into PVA films could enhance the UV-shielding efficacy by $13.3 \%$ in the ultraviolet spectrum $(250 \mathrm{~nm})$. The nanocomposite films with nano-lignin might offer a promising candidate for packaging and medicine.

\section{Conflicts of interest}

There are no conflicts to declare.

\section{Acknowledgements}

This work was supported by the National Natural Science Foundation of China (21406198) and Zhejiang Provincial Natural Science Foundation of China (LQ14B060003).

\section{Notes and references}

1 Z. Sun, B. Fridrich, A. de Santi, S. Elangovan and K. Barta, Chem. Rev., 2018, 118, 614-678.

2 M. Lievonen, J. J. Valle-Delgado, M.-L. Mattinen, E.-L. Hult, K. Lintinen, M. A. Kostiainen, A. Paananen, G. R. Szilvay, 
H. Setälä and M. Österberg, Green Chem., 2016, 18, 14161422.

3 A. A. Myint, H. W. Lee, B. Seo, W.-S. Son, J. Yoon, T. J. Yoon, H. J. Park, J. Yu, J. Yoon and Y.-W. Lee, Green Chem., 2016, 18, 2129-2146.

4 M. Norgren and H. Edlund, Curr. Opin. Colloid Interface Sci., 2014, 19, 409-416.

5 F. G. Calvo-Flores and J. A. Dobado, ChemSusChem, 2010, 3, 1227-1235.

6 S. V. Patil and D. S. Argyropoulos, ChemSusChem, 2017, 10, 3284-3303.

7 W. Yang, J. M. Kenny and D. Puglia, Ind. Crops Prod., 2015, 74, 348-356.

8 M. Fache, B. Boutevin and S. Caillol, Green Chem., 2016, 18, 712-725.

9 T. Saito, R. H. Brown, M. A. Hunt, D. L. Pickel, J. M. Pickel, J. M. Messman, F. S. Baker, M. Keller and A. K. Naskar, Green Chem., 2012, 14, 3295-3303.

10 C. D. Tran, J. Chen, J. K. Keum and A. K. Naskar, Adv. Funct. Mater., 2016, 26, 2677-2685.

11 M. Azadfar, A. H. Gao, M. V. Bule and S. Chen, Int. J. Biol. Macromol., 2015, 75, 58-66.

12 Y. Qian, X. Qiu, X. Zhong, D. Zhang, Y. Deng, D. Yang and S. Zhu, Ind. Eng. Chem. Res., 2015, 54, 12025-12030.

13 T. Sakaguchi, S. Nagano, M. Hara, S.-H. Hyon, M. Patel and K. Matsumura, Polym. J., 2017, 49, 535.

14 F. Xiong, Y. Wu, G. Li, Y. Han and F. Chu, Ind. Eng. Chem. Res., 2018, 57, 1207-1212.

15 B. Wang, D. Sun, H.-M. Wang, T.-Q. Yuan and R.-C. Sun, ACS Sustainable Chem. Eng., 2019, 7, 2658-2666.

16 C. Frangville, M. Rutkevičius, A. P. Richter, O. D. Velev, S. D. Stoyanov and V. N. Paunov, ChemPhysChem, 2012, 13, 4235-4243.

17 I. A. Gilca, V. I. Popa and C. Crestini, Ultrason. Sonochem., 2015, 23, 369-375.

18 M. Ago, S. Huan, M. Borghei, J. Raula, E. I. Kauppinen and O. J. Rojas, ACS Appl. Mater. Interfaces, 2016, 8, 23302-23310.

19 S. S. Nair, S. Sharma, Y. Pu, Q. Sun, S. Pan, J. Y. Zhu, Y. Deng and A. J. Ragauskas, ChemSusChem, 2014, 7, 3513-3520.

20 H. Zhao, J.-X. Wang, Q.-A. Wang, J.-F. Chen and J. Yun, Ind. Eng. Chem. Res., 2007, 46, 8229-8235.

21 Y. Qian, Y. Deng, X. Qiu, H. Li and D. Yang, Green Chem., 2014, 16, 2156-2163.

22 A. P. Richter, B. Bharti, H. B. Armstrong, J. S. Brown, D. Plemmons, V. N. Paunov, S. D. Stoyanov and O. D. Velev, Langmuir, 2016, 32, 6468-6477.

23 S. Badilescu and M. Packirisamy, Polymers, 2012, 4, 12781310.

24 R. C. S. Bicudo and M. H. A. Santana, Chem. Eng. Sci., 2012, 84, 134-141.

25 Q.-X. Zhang, L.-M. Xu, Y. Zhou, J.-X. Wang and J.-F. Chen, Ind. Eng. Chem. Res., 2011, 50, 13805-13812.

26 J. Ju, C. Zeng, L. Zhang and N. Xu, Chem. Eng. J., 2006, 116, 115-121.
27 A. Abou-Hassan, O. Sandre and V. Cabuil, Angew. Chem., Int. Ed., 2010, 49, 6268-6286.

28 K. Ren, Y. Chen and H. Wu, Curr. Opin. Biotechnol., 2014, 25, 78-85.

29 E. M. Chan, R. A. Mathies and A. P. Alivisatos, Nano Lett., 2003, 3, 199-201.

30 T. H. Eun, S.-H. Kim, W.-J. Jeong, S.-J. Jeon, S.-H. Kim and S.-M. Yang, Chem. Mater., 2009, 21, 201-203.

31 V. Sebastian Cabeza, S. Kuhn, A. A. Kulkarni and K. F. Jensen, Langmuir, 2012, 28, 7007-7013.

32 J. Parisi, L. Su and Y. Lei, Lab Chip, 2013, 13, 1501-1508.

33 A. Pongpeerapat, K. Itoh, Y. Tozuka, K. Moribe, T. Oguchi and K. Yamamoto, J. Drug Delivery Sci. Technol., 2004, 14, 441-447.

34 E. M. Abdelrazek, I. S. Elashmawi and S. Labeeb, Phys. B, 2010, 405, 2021-2027.

35 W. Zhao, L.-P. Xiao, G. Song, R.-C. Sun, L. He, S. Singh, B. A. Simmons and G. Cheng, Green Chem., 2017, 19, 32723281.

36 Y. Deng, X. Feng, M. Zhou, Y. Qian, H. Yu and X. Qiu, Biomacromolecules, 2011, 12, 1116-1125.

37 D. Tian, J. Hu, R. P. Chandra, J. N. Saddler and C. Lu, ACS Sustainable Chem. Eng., 2017, 5, 2702-2710.

38 K. C. Song, H. S. Lee, I. Y. Choung, K. I. Cho, Y. Ahn and E. J. Choi, Colloids Surf., A, 2006, 276, 162-167.

39 S. Karthika, T. K. Radhakrishnan and P. Kalaichelvi, Cryst. Growth Des., 2016, 16, 6663-6681.

40 G. S. Redner, C. G. Wagner, A. Baskaran and M. F. Hagan, Phys. Rev. Lett., 2016, 117, 148002.

41 A. Pongpeerapat, C. Wanawongthai, Y. Tozuka, K. Moribe and K. Yamamoto, Int. J. Pharm., 2008, 352, 309-316.

42 Y. Hou, J. Shao, Q. Fu, J. Li, J. Sun and Z. He, Int. J. Pharm., 2017, 516, 372-379.

43 S. Chatterjee, R. Prajapati, A. Bhattacharya and T. K. Mukherjee, Langmuir, 2014, 30, 9859-9865.

44 X.-Q. Hu, D.-Z. Ye, J.-B. Tang, L.-J. Zhang and X. Zhang, RSC $A d v .$, 2016, 6, 13797-13802.

45 L. Su, Z. Xing, D. Wang, G. Xu, S. Ren and G. Fang, BioResources, 2013, 8(3), 3532-3543.

46 X. Jiang, H. Li, Y. Luo, Y. Zhao and L. Hou, Int. J. Biol. Macromol., 2016, 82, 223-230.

47 N. Hameed, R. Xiong, N. V. Salim and Q. Guo, Cellulose, 2013, 20, 2517-2527.

48 H. Yang, R. Yan, H. Chen, D. H. Lee and C. Zheng, Fuel, 2007, 86, 1781-1788.

49 J. Qiao, J. Fu, R. Lin, J. Ma and J. Liu, Polymer, 2010, 51, 48504859.

50 H.-U. Ko, L. Zhai, J. H. Park, J. Y. Lee, D. Kim and J. Kim, J. Appl. Polym. Sci., 2018, 135, 46655.

51 G. Xu, S. Ren, D. Wang, L. Sun and G. Fang, BioResources, 2013, 8(2), 2510-2520.

52 F. Xiong, L. Zhou, L. Qian and S. Liu, BioResources, 2015, 10(1), 1149-1161. 